\title{
The Belgian Repository of Fundamental Atomic Data and Stellar Spectra (BRASS)
}

\author{
Alex Lobel ${ }^{1, *} \mathbb{0}$, Pierre Royer ${ }^{2}\left(\mathbb{0}\right.$, Christophe Martayan $^{3}$, Michael Laverick ${ }^{2}$, Thibault Merle ${ }^{4}$, \\ Mathieu Van der Swaelmen ${ }^{4}$, Peter A. M. van Hoof ${ }^{1}\left(\mathbb{D}\right.$, Marc David ${ }^{5}\left(\mathbb{D}\right.$ and Herman Hensberge ${ }^{1}$ \\ and Emmanuel Thienpont ${ }^{6}$ \\ 1 Royal Observatory of Belgium, Ringlaan 3, B-1180 Brussels, Belgium; p.vanhoof@oma.be (P.A.M.v.H.); \\ hhensberge@gmail.com (H.H.) \\ 2 Instituut voor Sterrenkunde, KU Leuven, Celestijnenlaan 200D, Box 2401, 3001 Leuven, Belgium; \\ pierre.royer@ster.kuleuven.be (P.R.); mike.laverick@kuleuven.be (M.L.) \\ 3 European Organisation for Astronomical Research in the Southern Hemisphere, Alonso de Córdova 3107, \\ Vitacura, 19001 Casilla, Santiago de Chile, Chile; cmartaya@eso.org \\ 4 Institut d'Astronomie et d'Astrophysique, Université Libre de Bruxelles, Av. Franklin Roosevelt 50, CP 226, \\ 1050 Brussels, Belgium; tmerle@ulb.ac.be (T.M.); mathieu@arcetri.inaf.it (M.V.d.S.) \\ 5 Onderzoeksgroep Toegepaste Wiskunde, Universiteit Antwerpen, Middelheimlaan 1, 2020 Antwerp, \\ Belgium; marc.david@uantwerpen.be \\ 6 Vereniging voor Sterrenkunde, Kapellebaan 56, 2811 Leest, Belgium; emmanuel.thienpont@gmail.com \\ * Correspondence: Alex.Lobel@oma.be
}

Received: 15 October 2019; Accepted: 19 November 2019; Published: 22 November 2019

check for updates

\begin{abstract}
Background: BRASS (Belgian Repository of Fundamental Atomic Data and Stellar Spectra) is an international networking project for the development of a new public database providing accurate fundamental atomic data of vital importance for stellar spectroscopic research. We present an overview of research results obtained in the past four years. Methods: The BRASS database offers atomic line data we thoroughly tested by comparing theoretical and observed stellar spectra. We perform extensive quality assessments of selected atomic input data using advanced radiative transfer spectrum synthesis calculations, which we compare to high-resolution Mercator-HERMES and ESO-VLT-UVES spectra of F-, G-, and K-type benchmark stars observed with very high signal-to-noise ratios. We have retrieved about half a million atomic lines required for our detailed spectrum synthesis calculations from the literature and online databases such as VAMDC, NIST, VALD, CHIANTI, Spectr-W ${ }^{3}$, TIPbase, TOPbase, SpectroWeb. Results: The atomic datasets have been cross-matched based on line electronic configuration information and organized in a new online repository called BRASS. The validated atomic data, combined with the observed and theoretical spectra are also interactively offered in BRASS. The combination of these datasets is a novel approach for its development providing a universal reference for advanced stellar spectroscopic research. Conclusion: We present an overview of the BRASS Data Interface developments allowing online user interaction for the combined spectrum and atomic data display, line identification, atomic data accuracy assessments including line $\log (g f)$-values, and line equivalent width measurements.
\end{abstract}

Keywords: quantitative stellar spectroscopy; spectral lines; atomic line data; atomic and spectral databases

\section{Introduction}

Fundamental atomic transition data, such as line oscillator strength values, are of central importance for determining the physical conditions in stellar atmospheres and for measuring their chemical compositions. Despite the significant work underway to produce these atomic data values 
for many astrophysically important ions, the uncertainties in these parameters remain large and can propagate throughout the entire field of astronomy. The Belgian repository of fundamental atomic data and stellar spectra (BRASS) aims to provide a large systematic and homogeneous quality assessment of the atomic data available for quantitative stellar spectroscopy. BRASS compares theoretical spectrum calculations to very high-quality observed spectra of FGK-type stars in order to critically evaluate the atomic data available for over a thousand atomic lines.

We report on the detailed analysis of six BRASS FGK-type benchmark HERMES spectra and the solar FTS spectrum. We present results of our quality assessments of atomic line oscillator strengths $(\log (g f)$-values) and line rest-wavelengths we have collected and combined in BRASS for advanced theoretical spectrum calculations of the benchmark spectra. Section 2.1 discusses the cross-matching of atomic transitions retrieved from a variety of atomic databases and the literature for the development of the Lines BRASS Data Interface. In Sections 2.2 and 2.3 we discuss the benchmark spectrum modeling results of 1091 investigated atomic lines for the $\log (g f)$ accuracy assessment pages we offer in the Spectra BRASS Data Interface. Section 2.4 provides a results comparison of two atomic data quality assessment methods used in BRASS. Section 2.5 presents a concise multiplet analysis of investigated Fe I transitions. The summary is provided in Section 3.

\section{BRASS Development Status}

\subsection{Lines BRASS Data Interface}

An important source of uncertainty in stellar spectrum synthesis calculations is the accuracy of atomic data of permitted transitions. It is crucial to constrain atomic data uncertainties for reliable measurements of the thermal conditions and chemical composition of stellar atmospheres. For BRASS we retrieve $\sim 400,000$ transition entries from various online atomic databases: VALD-3, NIST, Spectr-W ${ }^{3}$, TIPbase, TOPbase, CHIANTI, and SpectroWeb. We collect the atomic transition data of neutral species and ions up to $5^{+}$for wavelengths between 420 and $680 \mathrm{~nm}$. The datasets are homogenized and cross-matched against the BRASS atomic line list compilation. The BRASS list is composed of Kurucz and NIST V4.0 lines containing for each transition the species (element and ionization stage), line rest-wavelength, $\log (g f)$, upper and lower electronic configurations and energy levels, $J$-values, and the corresponding literature references. Our cross-matching is performed in two different ways: the parametric cross-match method is based on wavelength- and level energy-values for finding the same transition of a given species. On the other hand, the non-parametric cross-match method is based on detailed electronic configuration information for finding transitions that are physically identical between the datasets. The cross-matching accounts for atomic fine structure, the provided isotopic information, and the type of transition. It however does not account for currently missing hyperfine structure information.

The BRASS compilation was initially tested with theoretical spectrum calculations of the solar flux spectrum [1] and using Mercator-HERMES [2] spectra of selected B-, A-, F-, G-, and K-type stars (see [3]). The BRASS list has been also cleaned from numerous un-observed lines, spurious atomic \& molecular background features, and duplicated lines have been excluded. Note that the SpectroWeb atomic lines list was previously compiled from VALD-2 and NIST data (V2.0 through V4.0), and was also extensively tested similar to the BRASS list with theoretical spectrum calculations of high-quality hot and cool star spectra [4]. Table 1 lists the number of retrieved lines, source databases, dates of retrieval, and various atomic data values collected from each database. We have made extensive use of the online VAMDC portal offering homogenized datasets which has expedited the comparison and cross-matching of the datasets we have retrieved for BRASS. We partly incorporate data from TIPbase and TOPbase and include some of our expansions into fine-structure transitions [5]. We also calculate

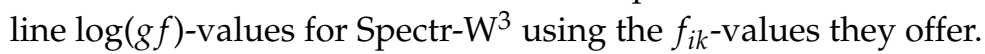


Table 1. Overview of the retrieved number of lines for BRASS from various atomic databases, including the retrieval dates and types of atomic data per source.

\begin{tabular}{|c|c|c|c|c|c|c|c|c|c|c|}
\hline Data Source & Origin & No. Lines & Date & Species & Line Wavelength & $A_{k i}$ & $f_{i k}$ & $\log (g f)$ & $E_{\text {low/up }}$ & $J_{\text {low/up }}$ \\
\hline BRASS & - & 82,337 & 2009-2012 & $\checkmark$ & $\checkmark$ & & & $\checkmark$ & $\checkmark$ & $\checkmark$ \\
\hline SpectroWeb & - & 62,181 & 2004-2008 & $\sqrt{3}$ & $\checkmark$ & & & & $\checkmark$ & \\
\hline VALD-3 & VALD & 158,861 & May 2016 & $\checkmark$ & $\checkmark$ & & & $\checkmark$ & $\checkmark$ & $\checkmark$ \\
\hline NIST & NIST & 36,123 & Mar 2016 & $\checkmark$ & $\checkmark$ & $\checkmark$ & & & $\checkmark$ & $\checkmark$ \\
\hline Spectr-W $W^{3}$ & VAMDC & 5515 & Mar 2016 & $\checkmark$ & $\checkmark$ & $\checkmark$ & $\checkmark$ & & $\checkmark$ & $\checkmark$ \\
\hline TIPbase & NORAD & 33,108 & Feb 2017 & $\checkmark$ & $\checkmark$ & $\checkmark$ & $\checkmark$ & & $\checkmark$ & \\
\hline TOPbase & VAMDC & 33,462 & May 2016 & $\checkmark$ & $\checkmark$ & & & $\checkmark$ & $\checkmark$ & \\
\hline CHIANTI & VAMDC & 3587 & Mar 2016 & $\checkmark$ & $\checkmark$ & $\checkmark$ & & $\checkmark$ & $\checkmark$ & $\checkmark$ \\
\hline
\end{tabular}

For the BRASS project [6] have used the non-parametric cross-match method to explore differences between multiple occurrences of identical transitions in the retrieved datasets. Detailed comparisons of $\lambda$ vs. $\Delta \lambda, E$ vs. $\Delta E, \Delta \lambda$ vs. $\Delta E$, and $\Delta \lambda$ vs. $\Delta \log (g f)$-values mainly reveal the presence of small-scale conversion precision differences. Large-scale systematic correlations are detected for a few cases only. However, the comparison of the line $\log (g f)$-values reveals differences in excess of 2 dex, which has important implications for quantitative stellar spectroscopy.

An investigation of duplicated transitions (also accounting for hyperfine-, isotopic-, and E2-M1 forbidden-transitions) in the retrieved datasets show a significant number of almost $2 \%$ in VALD-3 lists. These duplicates could be sourced back to the original work in $99 \%$ of cases, hence they were not produced by the databases from which the BRASS datasets are retrieved. The duplicated transitions for example have not been detected in the line datasets retrieved from NIST.

The cross-matched atomic datasets, including the BRASS atomic lines compilation, have been incorporated in the online Lines BRASS Data Interface (LBDI) at brass.sdf.org. Lists of duplicated lines are also offered there for a variety of data formats (HTML, ASCII, PDF). The left-hand panel of Figure 1 shows the LBDI that can be queried for a given element in a user-defined wavelength interval. In case a cross-match listing for every element is requested the users can set the Element input field to all. The query results can be sorted by increasing rest-wavelengths or $\sigma \log (g f)$ (standard deviation)-values marked in blue in Figure 2. The query results can be exported and saved in extensive line lists or per user-selected line to machine-readable (tab-separated ASCII) tables. Figure 2 shows for example the cross-matched atomic data of a S II line retrieved from seven atomic data sources providing five different $\log (g f)$-values ranging from -0.341 dex to -0.059 dex. The literature references of the $\log (g f)$-values are offered together with the upper and lower electronic configurations and level energies. The right-hand panel of Figure 1 also shows a subset of LBDI dynamic plots of the BRASS compilation $\log (g f)$-values vs. $\log (g f)$-difference values for VALD3-BRASS and NIST-BRASS of cross-matched Fe I lines and of Fe II lines. These dynamic plots can be interactively zoomed and the data of individual lines marked and displayed by mouse interaction. The $\log (g f)$-difference plots are provided per query for all atomic data sources and are ordered by neutral, singly, and multiply ionized species (from left to right). This provides users with an interactive and comprehensive overview of all cross-matched $\log (g f)$ datasets offered in BRASS. Note that the BRASS Data Interface also offers comprehensive Help pages (under the main green tab) for a number of BRASS usecases and corresponding tutorial videos. 


\section{Search BRASS database}

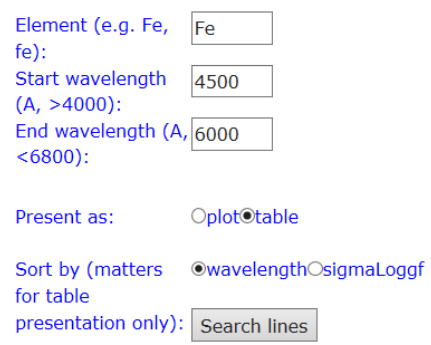

Fe 1
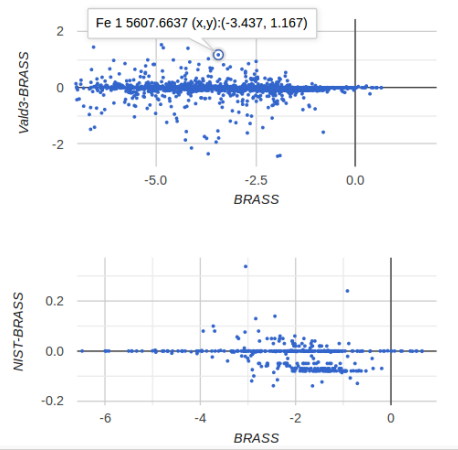

$\mathrm{Fe} 2$
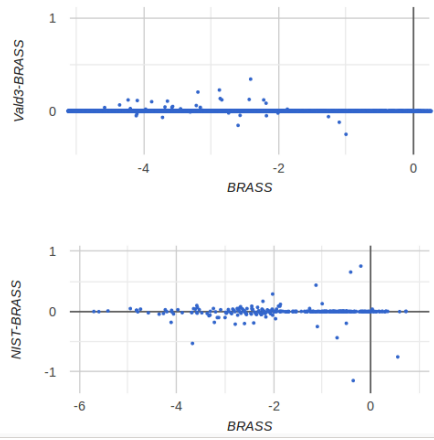

(a)

(b)

Figure 1. (a) The left-hand panel shows the Lines BRASS Data Interface query page. (b) The plot panels to the right show LBDI dynamic plots of BRASS $\log (g f)$-values compared to the $\log (g f)$-differences for VALD3-BRASS (top plot panels) and NIST-BRASS (bottom plot panels) of cross-matched Fe I and of Fe II lines (see text).

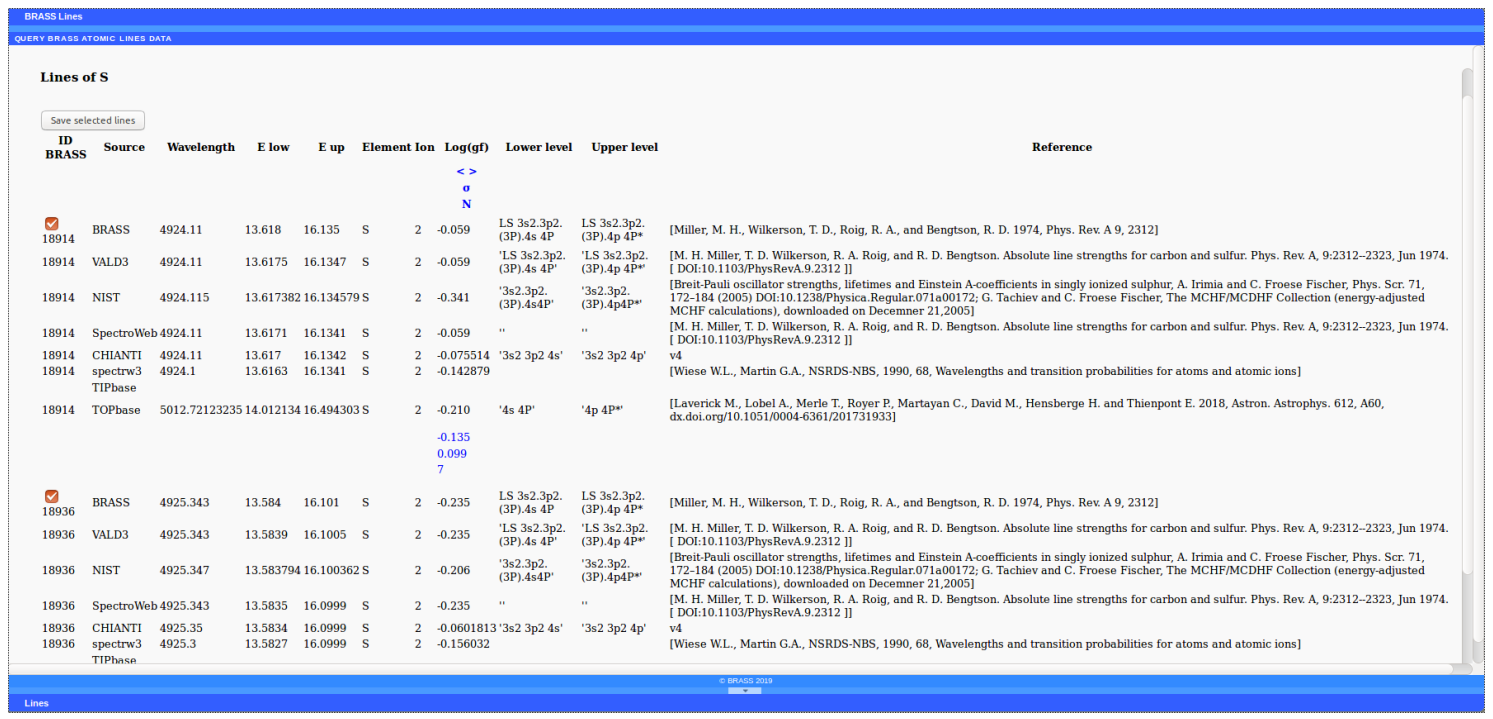

Figure 2. BRASS query results table for $S$ lines sorted by wavelength. The lines data are cross-matched with various atomic databases and the corresponding literature references are also offered.

\subsection{Spectra BRASS Data Interface}

For the BRASS project we observe benchmark spectra of a variety of bright stars $\left(V<7^{\mathrm{m}}\right)$ with the HERMES and ESO-VLT-UVES high-resolution spectrographs. We investigate HERMES benchmark spectra of 6 dwarf stars of F-, G-, and K spectral types observed with very high signal-to-noise ratios (SNR) of 800-1000: 51 Peg, 70 Oph, 70 Vir, 10 Tau, $\epsilon$ Eri, and $\beta$ Com ( $\mathrm{T}_{\text {eff }}$ between $5000 \mathrm{~K}$ and $6000 \mathrm{~K})$. The spectra are modelled in detail with advanced LTE synthesis calculations using 1-D hydrostatic atmosphere models (see [3]). The detailed spectrum modeling determines $T_{\text {eff- }}, \log (g)-$, $[\mathrm{M} / \mathrm{H}]-, \zeta_{\mu^{-}}, v \sin i-$, and $[\alpha / \mathrm{Fe}]-$ values we also compare to published stellar parameters measured with high-resolution spectra. The BRASS benchmark stars exclude binaries and are selected for non-variability and non-peculiarity. They are normal dwarf stars with narrow absorption lines having small rotational velocities below $6 \mathrm{~km} \mathrm{~s}^{-1}$ and metallicities very close to solar values. Metal-poor stars are excluded to avoid non-LTE effects in our theoretical spectrum calculations. 
The spectra of 11 BRASS benchmark stars (including the solar FTS spectrum) and the theoretical spectra are incorporated in the Spectra BRASS Data Interface (SBDI) shown in Figure 3. Users can interactively display up to four spectral Regions in two benchmark stars selected from the left-hand menus. The wavelengths of identified atomic lines are marked with red and blue labels. The red label numbers mark investigated lines. The red (and blue) labels can be clicked for displaying BRASS atomic data $\left(\log (g f)\right.$ - and $E_{l o w}$-values in a red 'graded list' in the right-hand panels), together with measured line properties such as the observed line equivalent widths ( $W_{\lambda}$ and $W_{\lambda}$-error), and the type of quality assessment we performed for the accuracy of the line $\log (g f)$ - and rest-wavelength -values (see Section 2.3). By double-clicking the red line labels users can build up lists of BRASS line data values (marked in green) for saving to their local computer disc. Clicking the 'View data quality' link in the red (or green) tables populates the 'Atomic Data Quality' tab in the central SBDI panel for a complete overview of the atomic line data quality assessment results BRASS offers for the investigated line.

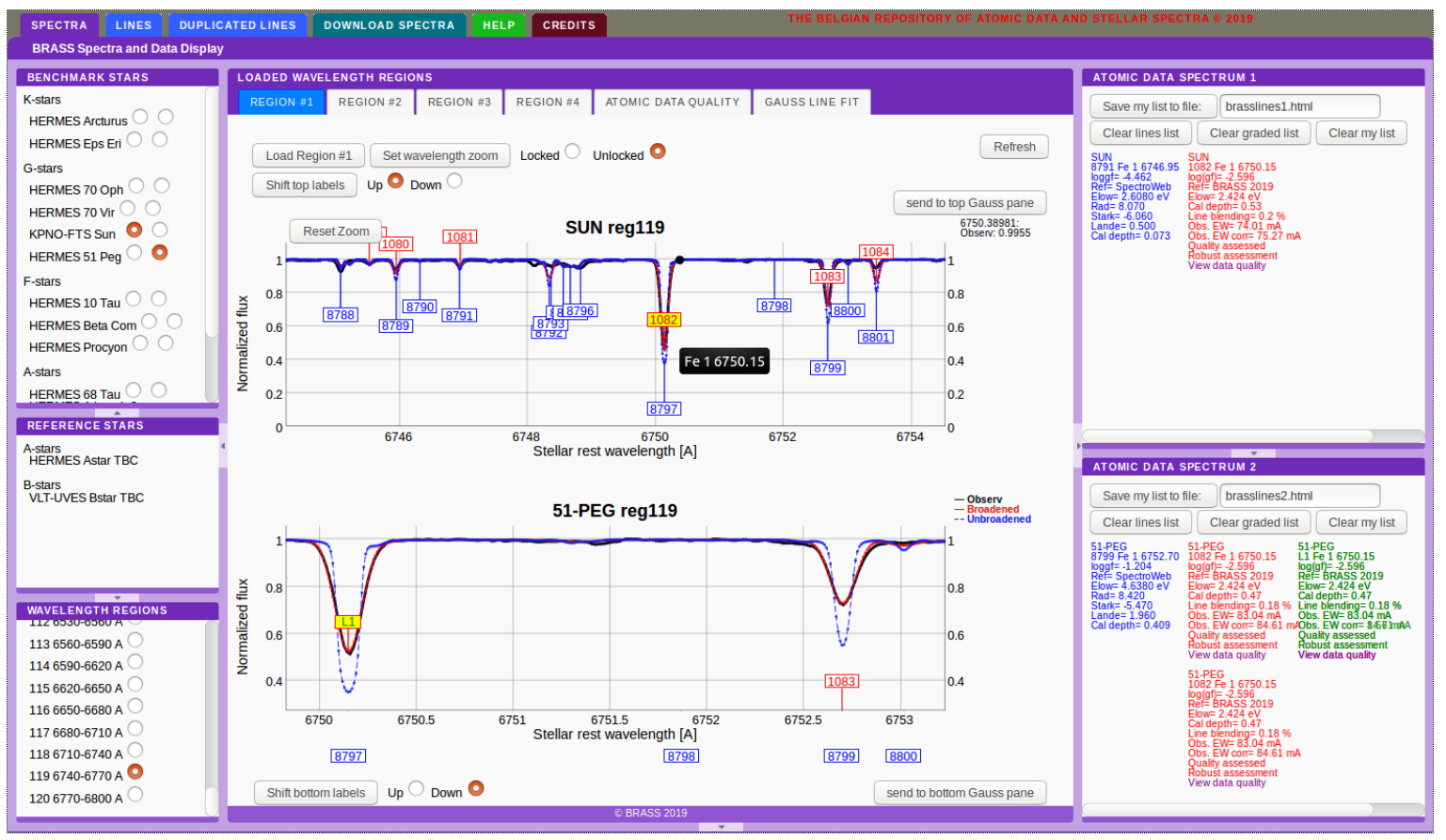

Figure 3. Screencopy of the SBDI for the solar FTS spectrum (central top panel) and the BRASS benchmark HERMES spectrum of the solar-like star 51 Peg (central bottom panel). The red and blue interactive labels mark identified absorption lines with atomic and line property data tables shown in the right-hand sub-panels. Atomic quality assessment pages are displayed under the central Atomic Data Quality tab by clicking on the View data quality link in the red or green line data tables.

\subsection{Atomic Line Data Quality Assessments}

A large-scale homogeneous selection of atomic lines was performed by [7] for BRASS by calculating the theoretical spectra of the 6 FGK benchmark HERMES spectra and the solar FTS spectrum. A selection of 1091 theoretically deep and sufficiently un-blended lines in the wavelength range $420 \mathrm{~nm}$ to $680 \mathrm{~nm}$ proved to be suitable for advanced quality assessments of the accuracy of the atomic datasets collected in BRASS. We determine astrophysical (semi-empiric) $\log (g f)$-values for these 1091 transitions using two commonly employed analysis methods. The agreement of the measured $\log (g f)$-values is used for selecting well-behaving lines for the quality assessment work. A total of 845 atomic lines are found to be suitable for quality assessment, of which 408 are robust against any systematic differences between both analysis methods. Around $53 \%$ of the quality-assessed lines are found to have at least one literature $\log (g f)$-value in agreement with the calculated values, although the remaining values can disagree by as much as 0.5 dex (see Section 2.4). 
For selecting atomic lines we calculate the amounts of blending in the 82337 BRASS lines of the solar and 51 Peg benchmark spectra. To reduce the impact of the line blending amounts on the atomic data quality assessment work a cut-off for blending of $\leq 10 \%$ is used, selected as a good balance between blending of the line core and the number of investigated lines. An additional cut-off on the central line core depth $\geq 0.02$ is also used to ensure the observed line profiles can be measured with sufficient accuracy. A total of 1515 atomic lines is initially selected as 'un-blended' lines in both stars. The lines selection procedure does not place limits on the atomic species. The equivalent line widths of the 1515 un-blended lines are automatically measured in all seven benchmark spectra using a single Gaussian fit profile. The line fit procedure is optimized using Gauss-Newton non-linear regression, or Nelder-Mead minimization in the case of slow convergence [8]. The best fit to the observed line fluxes is limited to the wavelength interval between two local flux maxima in both line wings exceeding $2 \%$ of the normalized continuum flux level. Beyond the local flux maxima the $W_{\lambda}$ integration is extended for Gaussian line wings. A goodness-of-fit value of $\chi^{2} \leq 0.95$ is used to remove poorly fitted (in addition to visual inspection), non-existent, too blended, or Earth line contaminated absorption features. The SBDI also offers an interactive $W_{\lambda}$ measurement tool under the Gauss line fit tab of the central panel in Figure 3. Users can select lines in observed BRASS spectra and display the single Gaussian best fit result together with the list of measured line properties for saving to their computer's disc.

The astrophysical $\log (g f)$-values are determined with two commonly employed methods. The measured line equivalent widths are converted into $\log (g f)$-values using the theoretical curve-of-growth calculated for the line in each benchmark star (called COG method). The other method varies the $\log (g f)$-values in detailed radiative transfer calculations for determining the best-fit value to the observed line profile. The latter method is called GRID because it involves an iterative line modeling procedure for which a grid of spectra is calculated and the best fitting spectrum for a range of $\log (g f)$ - and $\lambda$-values is obtained with $\chi^{2}$-minimization by interpolating in steps of $\Delta \lambda(0.005 \AA)$ and $\Delta \log (g f)(0.01 \mathrm{dex})$ using a bivariate cubic spline fit. Both methods introduce assessable uncertainties resulting from the accuracy of the best fit procedures to the observed $W_{\lambda}$-value and the continuum normalized line flux distribution. The uncertainties can be attributed to the spectral SNR, specific atmosphere modeling assumptions, the continuum flux level normalization procedure, and blending with the observed line unaccounted for in our theoretical spectrum calculations. For the seven BRASS benchmark spectra we measure an intrinsic scatter between both methods of \pm 0.04 dex ( $1 \sigma$ standard deviation) for line blending levels below $3-4 \%$. The value of \pm 0.04 dex is therefore used as a constraint on the lines selection for limiting the impact of systematic differences between both methods on the atomic data quality assessment results. In our analysis method the close agreement between the COG and GRID $\log (g f)$-values is required for quality assessing the literature $\log (g f)$-values retrieved for BRASS. The COG $\log (g f)$-value is calculated for a given transition with the observed $W_{\lambda}$-value of an absorption feature we can attribute to the line, while the GRID $\log (g f)$-value results from complete theoretical spectrum calculations that fit the observed spectrum incorporating the (sufficiently un-blended) line profile.

The SBDI offers atomic data quality assessment pages showing plots and data values for the 1091 investigated lines. Figure 4 shows a screencopy of the SBDI Atomic Data Quality tab for the Ni I $\lambda 6598$ line observed in the BRASS benchmark spectra (solid black line with dots) over-plotted with the theoretical profiles we calculate for the atomic data values retrieved from four atomic databases. The line profiles calculated for $\log (g f)$-values we determine from the GRID and COG analysis methods are over-plotted in blue and green colors, respectively. Users can interactively zoom-in, pan, and reset these line profile plots for each benchmark star. By clicking the check boxes above the plots the theoretical line profiles calculated with the $\log (g f)$-values in the atomic databases are also over-plotted for user inspection. The Quality assessment table shown below the line profile plots lists the GRID and COG line $\log (g f)$ - and rest-wavelength $(\lambda)$-values, together with the differences $(\Delta \log (g f)$ and $\Delta \lambda)$ with respect to the GRID values. The last column of this table offers a Yes/No flag indicating if 
the $\Delta \log (g f)$-value is within the errors of the GRID $\log (g f)$-value. The flags and $\Delta$-values are useful for determining if the $\log (g f)$-values retrieved from the databases for BRASS are sufficiently accurate for detailed spectrum synthesis calculations. For example, for Ni I $\lambda 6598$ we determine GRID $\log (g f)$ and COG $\log (g f)$-values within errors of each other (hence having quality-assessable atomic data), but not within \pm 0.04 dex of each other, signaling the line is not robust against the analysis method. The bottom table with Equivalent widths offers the observed (Measured) and theoretical $W_{\lambda}$-values (in $\mathrm{m} \AA$ ) we calculate for the investigated line per database in all the benchmark stars. Note that we also add small corrections listed for $\Delta W_{\lambda}^{\text {corr }}$ to the observed line equivalent width values in case the line saturates on the curve-of-growth and Voigt profile corrections are introduced in our best Gaussian line fit procedure.

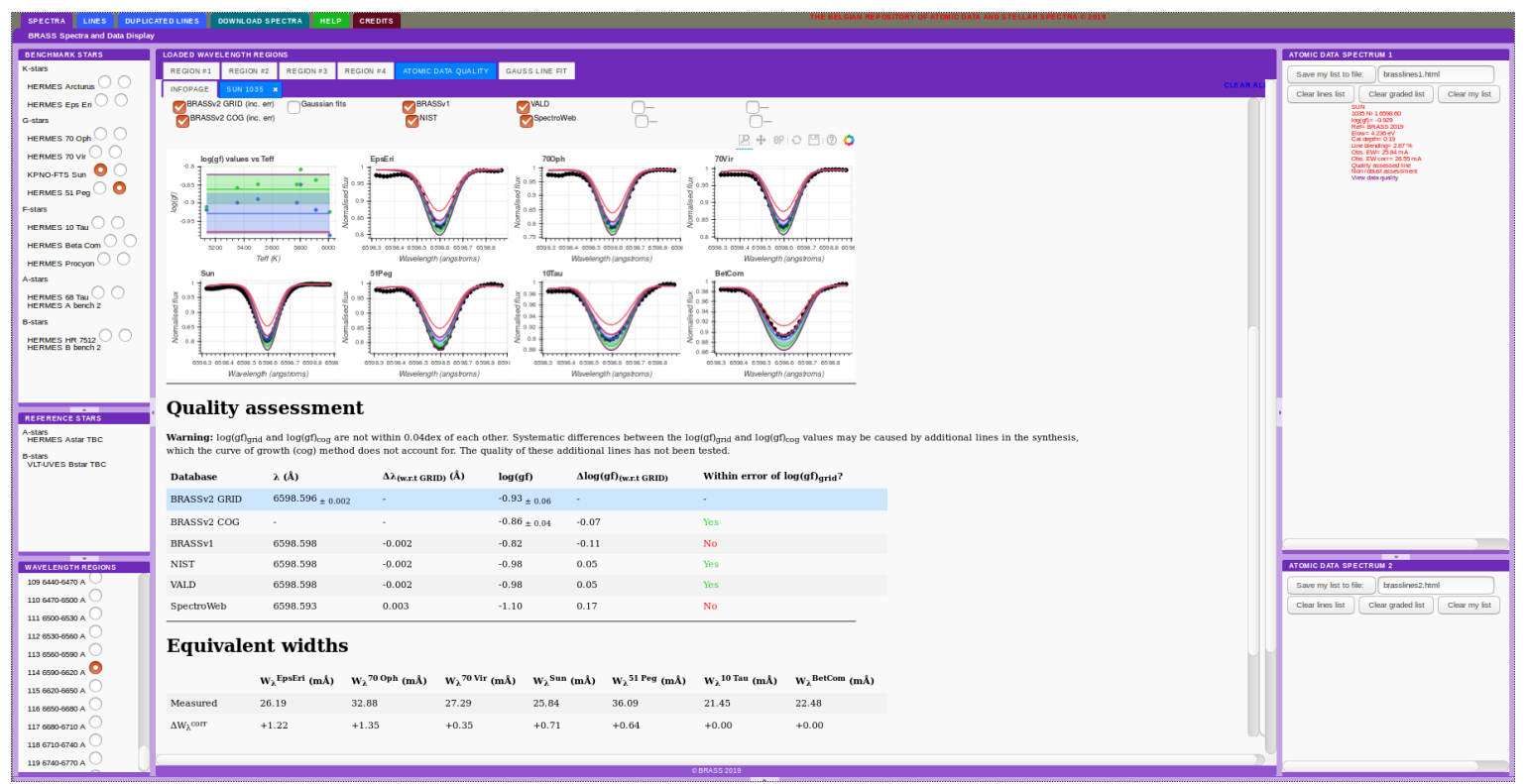

Figure 4. Screencopy of the SBDI page for the atomic data quality assessment results of the Ni I $\lambda 6598$ line observed in seven BRASS benchmark spectra. The observed and theoretical line profiles shown in the sub-panels can be displayed with user interaction. The SBDI pages offer an overview of all atomic data quality results for each investigated line together with the observed and theoretical line equivalent width values (see text).

\subsection{Comparison of Atomic Data Quality Assessment Results}

We find 845 of the 1091 investigated lines to be quality-assessable, and 408 are also analysis-independent lines. Nearly half of the investigated and quality-assessable lines are of Fe I, while another $\sim 10 \%$ belong to singly ionized species. The retrieved literature $\log (g f)$-values of a quality-assessable line are considered in agreement with our results and can be recommended in theoretical spectrum calculations only in case they agree within the errors of the mean (averaged over all benchmarks) GRID $\log (g f)$-value and its standard deviation. We do not consider any literature errorbars because they are not available for the vast majority of investigated lines. In most cases we adopt the mean GRID $\log (g f)$-value as the BRASS reference value because the GRID method yields smaller $\chi^{2}$-values than the COG method. About $53 \%$ of the quality-assessable lines have literature $\log (g f)$-values in agreement with the mean GRID $\log (g f)$-values. A similar percentage of the 408 analysis-independent lines have sufficiently accurate atomic data. The majority of Fe-group species (V I, Cr I, Mn I, Co I, Ni I, Ti I, and Sc II, Ti II, Fe II) have a good number of lines with accurate atomic data for $70-75 \%$ of the lines. The Fe I lines, however, have only $\sim 38 \%$ with sufficiently accurate atomic data (see Section 2.5).

The right-hand panel of Figure 5 shows mean GRID $\log (g f)$ - (blue dots) and mean COG $\log (g f)$-values (black dots) compared to the $\log (g f)$-values in the BRASS (input) dataset for the 
408 analysis-independent lines (where both COG and GRID astrophysical values agree within \pm 0.04 dex). We find sizable differences with the BRASS $\log (g f)$-values for a considerable number of lines. Difference $\log (g f)$-values in excess of \pm 0.5 dex are observed. The inset panel shows lines with smaller $\log (g f)$ differences $(\leq 0.2 \mathrm{dex})$, although many are not in agreement within the derived errorbars.

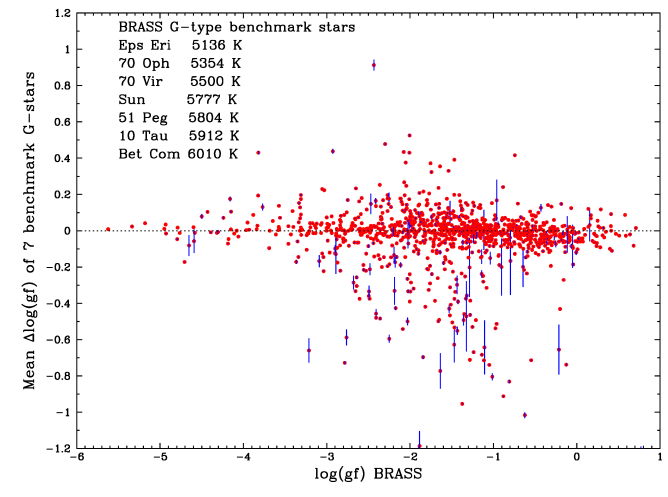

(a)

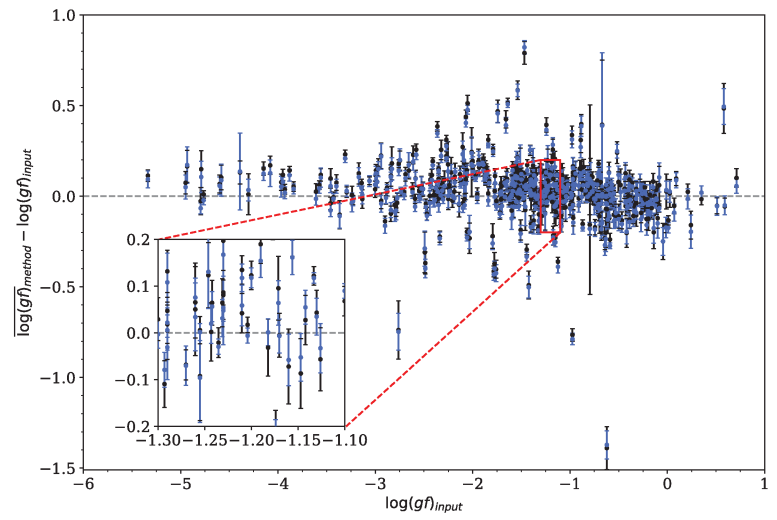

(b)

Figure 5. (a) The left-hand panel shows mean $\Delta \log (g f)$-values (red dots) determined with the linear approximation method against the literature $\log (g f)$-values retrieved for BRASS. The standard deviations (blue lines) for the 7 benchmark stars stay below 0.02 dex for the vast majority of investigated lines. (b) The right-hand panel shows a similar difference plot for astrophysical $\log (g f)$-values of 408 analysis-independent lines from the GRID (blue dots) vs. COG (black dots) atomic data quality assessment method (see text).

It is important to point out that the large differences between the literature $\log (g f)$-values we retrieve for BRASS and the mean astrophysical $\log (g f)$-values calculated with FGK BRASS benchmark spectra are also detected using a linear approximation method. Absorption lines on the linear part of the curve-of-growth follow a linear relationship between $W_{\lambda}$ - and $\log (g f)$-values. For these lines the difference between observed and theoretical $\log (g f)$-values equals $\log \left(W_{\lambda}^{\text {obs }} / W_{\lambda}^{\text {mod }}\right)$, where $W_{\lambda}^{\text {mod }}$ is the line equivalent width we calculate with the theoretical $\log (g f)$-value.

The left-hand panel of Figure 5 shows the mean of the $\Delta \log (g f)$-values we calculate for the seven BRASS benchmark stars against the retrieved BRASS $\log (g f)$-values. The largest mean $\Delta \log (g f)$-values can also exceed $0.5 \mathrm{dex}$, although the standard deviations are $\leq 0.02$ dex for the majority of investigated lines (blue errorbars) (see [8]). Note however that the mean COG and GRID errors are 0.065 dex and 0.05 dex, respectively, or about 3 times larger. Similar to the GRID vs. COG quality assessment method the mean $\log (g f)$-differences we calculate with this linear approximation method remain typically below \pm 1 dex and are chiefly observed for the medium-strong lines having $-3 \leq \log (g f)$ $\leq-0.5$. The lines with negative $\log (g f)$-differences were also found in a separate analysis of Fe-group element lines in the solar FTS spectrum and in HERMES and UVES spectra of Procyon and $\epsilon$ Eri [4]. For these lines the literature $\log (g f)$-values are overestimated yielding theoretical $W_{\lambda}$-values that exceed observed values. Similar to the full-fledged GRID vs. COG analysis method smaller $\Delta \log (g f)$-values are also found towards the weakest $(\log (g f)<-3.5)$ and strongest $(\log (g f)>0)$ investigated lines.

\subsection{Multiplet Analysis of Fe I Transitions in BRASS}

The rather small percentage of only $\sim 38 \%$ of sufficiently accurate atomic data for the Fe I lines in BRASS calls for an investigation of its origin we briefly discuss. The left-hand panel of Figure 6 shows the curve-of-growth for Fe I lines we observe in the solar benchmark spectrum. The black dots show observed (reduced) $W_{\lambda} / \lambda$-values against $\log (g f)$ (co-added with other terms), for $\log (g f)$-values 
in the (input) BRASS compilation of Table 1. We find considerable scatter for the transitions on the linear part of the curve or mainly for the weak and medium-strong Fe I lines. The large scatter is due to the limited accuracy of the literature $\log (g f)$-values for these lines. We find that this scatter across the curve substantially reduces after replacing the literature $\log (g f)$-values with the ones we calculate from the linear approximation method in Section 2.4 shown with red dots. By replacing the $\log (g f)$-values with the ones we calculate from the COG vs. GRID method the scatter nearly vanishes and the curve assumes the smooth (and narrow) shape required for atomic lines belonging to the same species in stellar spectra. The large percentage we find of over $60 \%$ of literature Fe I atomic data with limited quality mainly results from medium-strong (and weak) lines having $-3 \leq \log (g f) \leq-0.5$ in Figure 5.

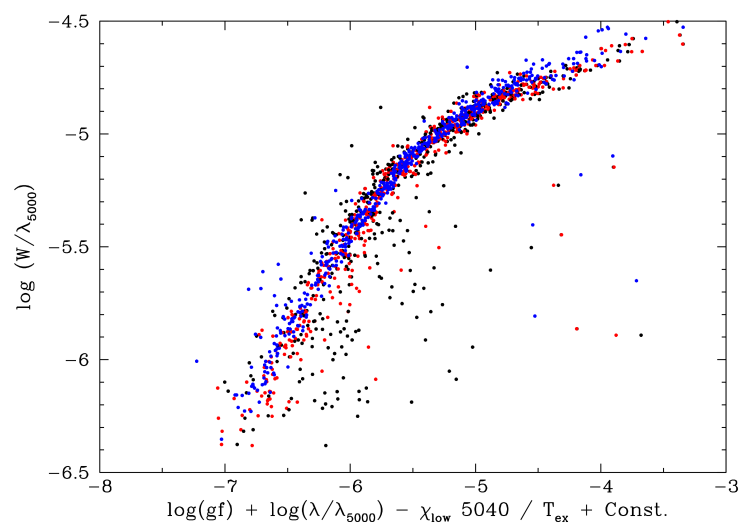

(a)

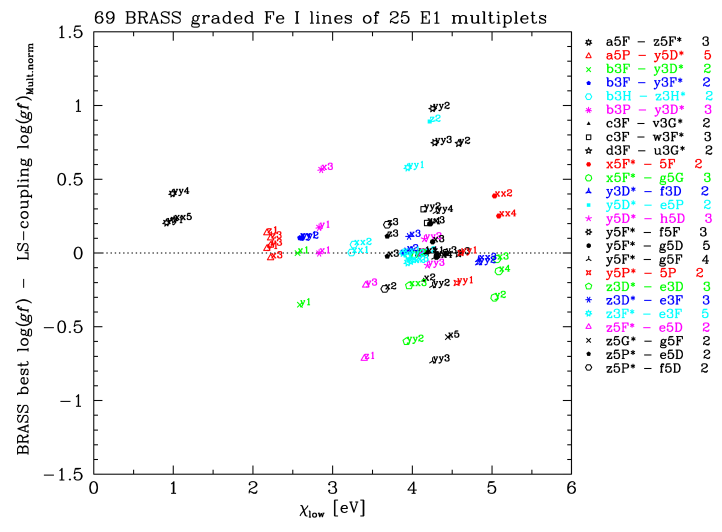

(b)

Figure 6. (a) The left-hand panel shows the curve-of-growth of Fe I lines investigated for BRASS. Substantial scatter observed in medium-strong and weak lines using literature $\log (g f)$-values (black dots) is properly removed by replacing them with the values of the linear approximation method (red dots) or the GRID $\log (g f)$-values (blue dots). (b) The right-hand panel shows the increase in $\Delta \log (g f)$-values (BRASS-LS) against $E_{\text {low }}$ for lines of 25 multiplets (also marked to the right for the number of lines) having literature retrieved $\log (g f)$-values of lesser quality in case $E_{\text {low }}>4 \mathrm{eV}$.

A more extensive analysis of the Fe I fine structure data we retrieve for BRASS reveals that the lines with limited/poor $\log (g f)$ quality mostly have $E_{\text {low }}>4 \mathrm{eV}$. We compile 25 electric dipole multiplets of 69 Fe I lines in BRASS, also shown in the right-hand panel of Figure 6. For each line of these multiplets we calculate the relative line strength ratios assuming single-configuration Russell-Saunders (LS) coupling and obeying the selection rules for these permitted transitions. The calculated multiplet line strengths (using Wigner 6j-symbol calculations) are normalized by scaling the LS-coupling $\log (g f)$-values to the strongest available principal line (marked with $\mathrm{x}_{1}$ or $\mathrm{x}_{2}$ in Figure 6), or the largest $\log (g f)$-value we calculate with the GRID method using the benchmark spectra. For a number of multiplets we find reasonable to good agreement between the LS and GRID relative $\log (g f)$-values. For example multiplet $a 5 P \rightarrow y 5 D\left(E_{\text {low }}=2.17-2.23 \mathrm{eV}\right)$ shows very similar distributions across its principal $(x)$ and satellite $(y$ and $z)$ transitions. For multiplet $y 3 D \rightarrow y 3 F\left(E_{l o w}=4.73-4.84 \mathrm{eV}\right)$ the relative $\log (g f)$ distributions agree less, but also show differences between the literature and GRID $\log (g f)$-values of $\sim 1.0 \mathrm{dex}$. This is also the case for $x 5 F \rightarrow 5 F\left(E_{\text {low }}=4.9-5.1 \mathrm{eV}\right)$ and $y 5 D \rightarrow e 5 P$ $\left(E_{\text {low }}=4.1-4.23 \mathrm{eV}\right)$ multiplets for which the relative LS and GRID $\log (g f)$-distributions across the $x$, $y$, and $z$ line series are dissimilar and a re-normalization cannot remove the large differences above 0.5-1.0 dex for individual lines. The right-hand panel of Figure 6 shows that the differences between the normalized LS and the (best) BRASS GRID $\log (g f)$-values increase towards larger $E_{l o w}$-values. 
For lines having $E_{\text {low }}>4 \mathrm{eV}$ in the 25 studied multiplets the differences can increase above 0.5-1.0 dex mainly for the satellite transitions (marked $y$ ).

Using the interactive NIST Grotrian diagrams we find that both the $y 5 D$ and $x 5 F$ multiplet energy levels have level components that lie very close in energy to neighboring high energy levels of other atomic terms. For example the $y 5 D(J=1)$ level at $4.217 \mathrm{eV}$ falls next to another energy level at $4.220 \mathrm{eV}$ corresponding to the $y 5 F(J=5)$ level. In addition, the $x 5 F(J=2)$ level at $5.085 \mathrm{eV}$ lies near a $z 5 S(J$ $=2$ ) level at $5.070 \mathrm{eV}$. The proximity of other nearby energy levels for these multiplet lower levels yields significant configuration interaction between the levels. The LS-coupling calculations cannot accurately predict the relative line strengths assuming single electronic configuration interaction. The increasing differences between the literature, LS and GRID $\log (g f)$-values towards larger $E_{\text {low }}$ results from inaccurate theoretical Fe I $\log (g f)$-values due to poorly constrained configuration mixing coefficients and inaccurate or incomplete theoretical energy levels for the large number of close energy levels above $\sim 4 \mathrm{eV}$ in the neutral Fe atom.

\section{Summary}

This paper presents new progress results for BRASS. We present new quality assessment results for the accuracy of atomic $\log (g f)$-values required for theoretical modeling of high-resolution stellar spectra using 7 FGK-type benchmark stars including the Sun. Astrophysical $\log (g f)$-values have been calculated for 1091 carefully selected sufficiently un-blended line transitions between $420 \mathrm{~nm}$ and $680 \mathrm{~nm}$ using two commonly employed methods. The agreement between both methods selects 845 lines suitable for our quality-assessments. An investigation of mean $\Delta \log (g f)$-values reveals large differences for lines with limited atomic data quality offered in the literature for $-3 \leq \log (g f) \leq-0.5$. We find that $\sim 53 \%$ of the quality-assessable lines have at least one literature $\log (g f)$-value in agreement with the astrophysical values, while values for other lines can differ by more than 0.5 dex. Only $~ 38 \%$ of the investigated Fe I lines have sufficiently accurate literature $\log (g f)$-values, while $\sim 70-75 \%$ for other Fe-group element lines. The large percentage we find for theoretical Fe I $\log (g f)$-values of low quality offered in the literature mainly results from medium-strong and weak lines in multiplets with $E_{\text {low }}>4 \mathrm{eV}$, likely due to strong level mixing and inaccurate/incomplete energy levels. We also find that the majority of $\Delta \lambda$-values are below $\pm 0.01 \AA$, comparable to the high accuracy of the HERMES wavelength scale.

The Lines and Spectra BRASS Data Interface have been updated with the cross-matched atomic datasets and observed and theoretical stellar spectra. Users of the BRASS repository can query the LBDI for atomic data downloading, including the corresponding literature references, and the interactive display of dynamic plots for comparisons of database $\log (g f)$-values. The SBDI offers interactive display tools for the (observed and theoretical) benchmark spectra, combined with line identifications and atomic data values and line properties for user downloading. The BRASS repository offers interactive atomic data quality assessment pages for the 1091 investigated spectral lines. It also offers tools for interactive line equivalent width measurements and comprehensive help pages and tutorial videos to its users.

Author Contributions: BRASS conceptualization, A.L., P.R. and C.M.; methodology, A.L and M.L.; software development, A.L., M.L, and E.T.; results validation, A.L., M.L., P.R., M.D. and H.H.; project investigations, A.L., C.M., M.L., T.M., M.V.d.S. and P.A.M.v.H.; database resources, A.L.; data curation, A.L., M.L., C.M., T.M., and M.V.d.S.; writing —original draft preparation, A.L.; database visualization, A.L. and E.T.; supervision, A.L., M.D., H.H. and E.T.; project coordination, A.L.; project administration, A.L.; funding acquisition, A.L., P.R. and C.M.

Funding: The research for the present results has been subsidised by the Belgian Federal Science policy Office under contract No. BR/143/A2/BRASS.

Acknowledgments: The BRASS Team would like to thank everybody who directly or indirectly has contributed since 2015 to the realization of the BRASS project.

Conflicts of Interest: The authors declare no conflict of interest. 


\section{Abbreviations}

The following abbreviations are used in this manuscript:

$\begin{array}{ll}\text { LBDI } & \text { Lines BRASS Data Interface } \\ \text { SBDI } & \text { Spectra BRASS Data Interface } \\ \text { NIST } & \text { National Institute of Standards and Technology } \\ \text { VALD } & \text { Vienna Atomic Line Database } \\ \text { VAMDC } & \text { Virtual Atomic and Molecular Data Centre } \\ \text { TIPbase } & \text { The Iron Project database } \\ \text { TOPbase } & \text { The Opacity Project database } \\ \text { CHIANTI } & \text { The CHIANTI atomic database } \\ \text { SpectroWeb } & \text { The SpectroWeb database } \\ \text { Spectr-W } & \text { The Spectr-W }{ }^{3} \text { database } \\ \text { HERMES } & \text { High Efficiency and Resolution Mercator Echelle Spectrograph } \\ \text { FTS } & \text { Fourier Transform Spectrograph } \\ \text { SNR } & \text { Signal to Noise Ratio } \\ \text { FWHM } & \text { Full-Width Half-Maximum }\end{array}$

\section{References}

1. Neckel, H.; Labs, D. The solar radiation between 3300 and 12500 Å. Sol. Phys. 1984, 90, 205. [CrossRef]

2. Raskin, G.; Van Winckel, H.; Hensberge, H.; Jorissen, A.; Lehmann, H.; Waelkens, C.; Avila, G.; De Cuyper, J.P.; Degroote, P.; Dubosson, R.; et al. HERMES: A high-resolution fibre-fed spectrograph for the Mercator telescope. Astron. Astrophys. 2011, 526, A69. [CrossRef]

3. Lobel, A.; Royer, P.; Martayan, C.; Laverick, M.; Merle, T.; David, M.; Hensberge, H.; Thienpont, E. The Belgian repository of fundamental atomic data and stellar spectra. Can. J. Phys. 2017. [CrossRef]

4. Lobel, A. Oscillator Strength Measurements of Atomic Absorption Lines from Stellar Spectra. Can. J. Phys. 2011, 89, 395-402. [CrossRef]

5. Merle, T.; Laverick, M.; Lobel, A.; Royer, P.; Van der Swaelmen, M.; Frémat, Y.; Sekaran, S.; Martayan, C.; van Hoof, P.; David, M.; et al. The Belgian Repository of fundamental Atomic data and Stellar Spectra (BRASS). In Proc. of Semaine de l'Astrophysique Francaise 2018; Di Matteo, P., Billebaud, F., Herpin, F., Lagarde, N., Marquette, J.-B., Robin, A., Venot, O., Eds.; Société Francaise d'Astronomie et d'Astrophysique (SF2A): Bordeaux, France, 2018; p. 153.

6. Laverick, M.; Lobel, A.; Merle, T.; Royer, P.; Martayan, C.; David, M.; Hensberge, H.; Thienpont, E. The Belgian repository of fundamental atomic data and stellar spectra (BRASS) I. Cross-matching atomic databases of astrophysical interest. Astron. Astrophys. 2018, 612, A60. [CrossRef]

7. Laverick, M.; Lobel, A.; Royer, P.; Merle, T.; Martayan, C.; van Hoof, P.; Van der Swaelmen, M.; David, M.; Hensberge, H.; Thienpont, E. The Belgian repository of fundamental atomic data and stellar spectra (BRASS) II. Quality assessment of atomic data for unblended lines in FGK stars. Astron. Astrophys. 2019, 624, A60. [CrossRef]

8. Lobel, A.; Royer, P.; Martayan, C.; Laverick, M.; Merle, T.; van Hoof, P.A.M.; Van der Swaelmen, M.; David, M.; Hensberge, H.; Thienpont, E. The Belgian Repository of Fundamental Atomic Data and Stellar Spectra: Atomic Line Data Validation. In Workshop on Astrophysical Opacities; Mendoza, C., Turck-Chiéze, S., Colgan, J., Eds.; Astron. Soc. of the Pacific CS: San Francisco, CA, USA, 2018; Volume 515, pp. 255-262. 